\title{
Editorial
}

\section{Advanced Materials and Nanotechnology for Sustainable Energy Development}

\author{
Ziyang Huo, ${ }^{1}$ Cheng Hao Wu, ${ }^{2}$ Zhongwei Zhu, $^{3}$ and Yuxin Zhao ${ }^{4}$ \\ ${ }^{1}$ Griffith University, Brisbane, QLD 4109, Australia \\ ${ }^{2}$ Lawrence Berkeley National Laboratory, Berkeley, CA 94720, USA \\ ${ }^{3}$ Department of Chemistry, University of California, Berkeley, CA 94720, USA \\ ${ }^{4}$ SINOPEC Safety Engineering Institute, Qingdao 266071, China \\ Correspondence should be addressed to Ziyang Huo; ziyanghuo@gmail.com \\ Received 8 October 2015; Accepted 16 November 2015 \\ Copyright (C) 2015 Ziyang Huo et al. This is an open access article distributed under the Creative Commons Attribution License, \\ which permits unrestricted use, distribution, and reproduction in any medium, provided the original work is properly cited.
}

Energy is the material foundation of human activities and also the single most valuable resource for the production activities of human society. Materials play a pivotal role in advancing technologies that can offer efficient renewable energy solutions for the future. This special issue has been established as an international foremost interdisciplinary forum that aims to publish high quality and original full research articles on all aspects of the study of materials for the deployment of renewable and sustainable energy technologies. The special issue covers experimental and theoretical aspects of materials and prototype devices for sustainable energy conversion, storage, and saving, together with materials needed for renewable energy production. It brings together stakeholders from universities, industries, government agents, and businesses that are involved in the invention, design, development, and implementation of sustainable technologies.

The research work has already been published in this special issue which discusses comprehensive technologies for wastewater treatment, strategies for controlling gaseous pollutant releases within chemical plant, evaluation of FCC catalysis poisoning mechanism, clean technologies for fossil fuel use, new-type photocatalysis material design with controllable morphology for solar energy conversion, and so forth. These studies describe important, intriguing, and systematic investigations on advanced materials and technologies for dealing with the key technologies and important issues that continue to haunt the global energy industry. They also tie together many aspects of current energy transportation science and technology, exhibiting outstanding industrial insights that have the potential to encourage and stimulate fresh perspectives on challenges, opportunities, and solutions to energy and environmental sustainability.

Ziyang Huo Cheng Hao Wu Zhongwei Zhu Yuxin Zhao 

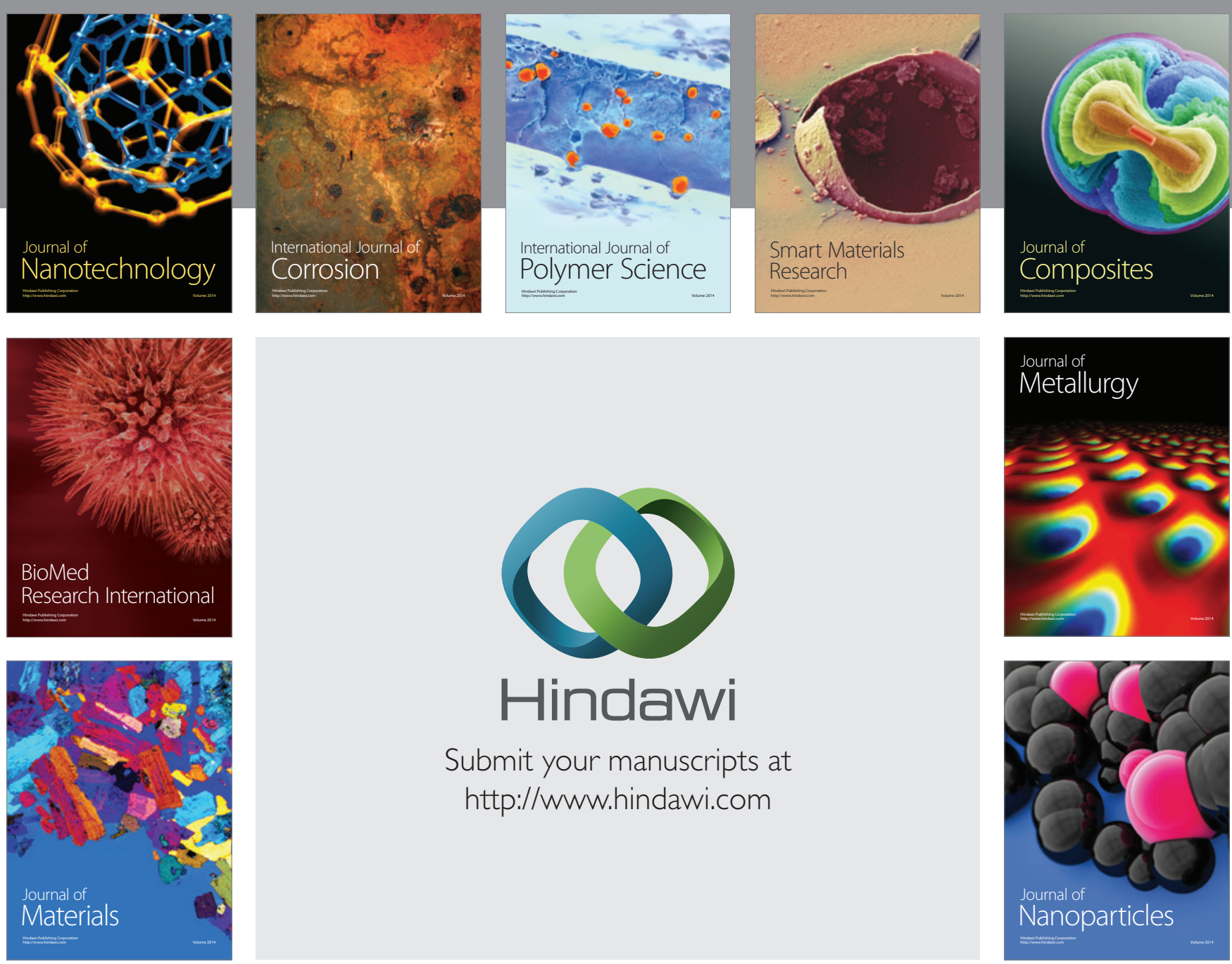

Submit your manuscripts at http://www.hindawi.com
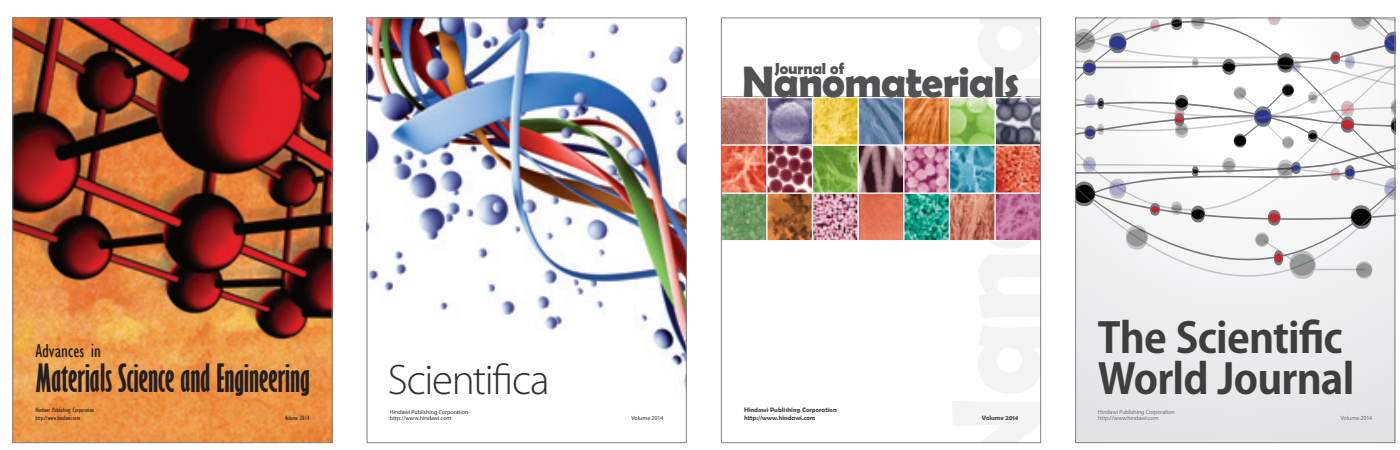

\section{The Scientific World Journal}
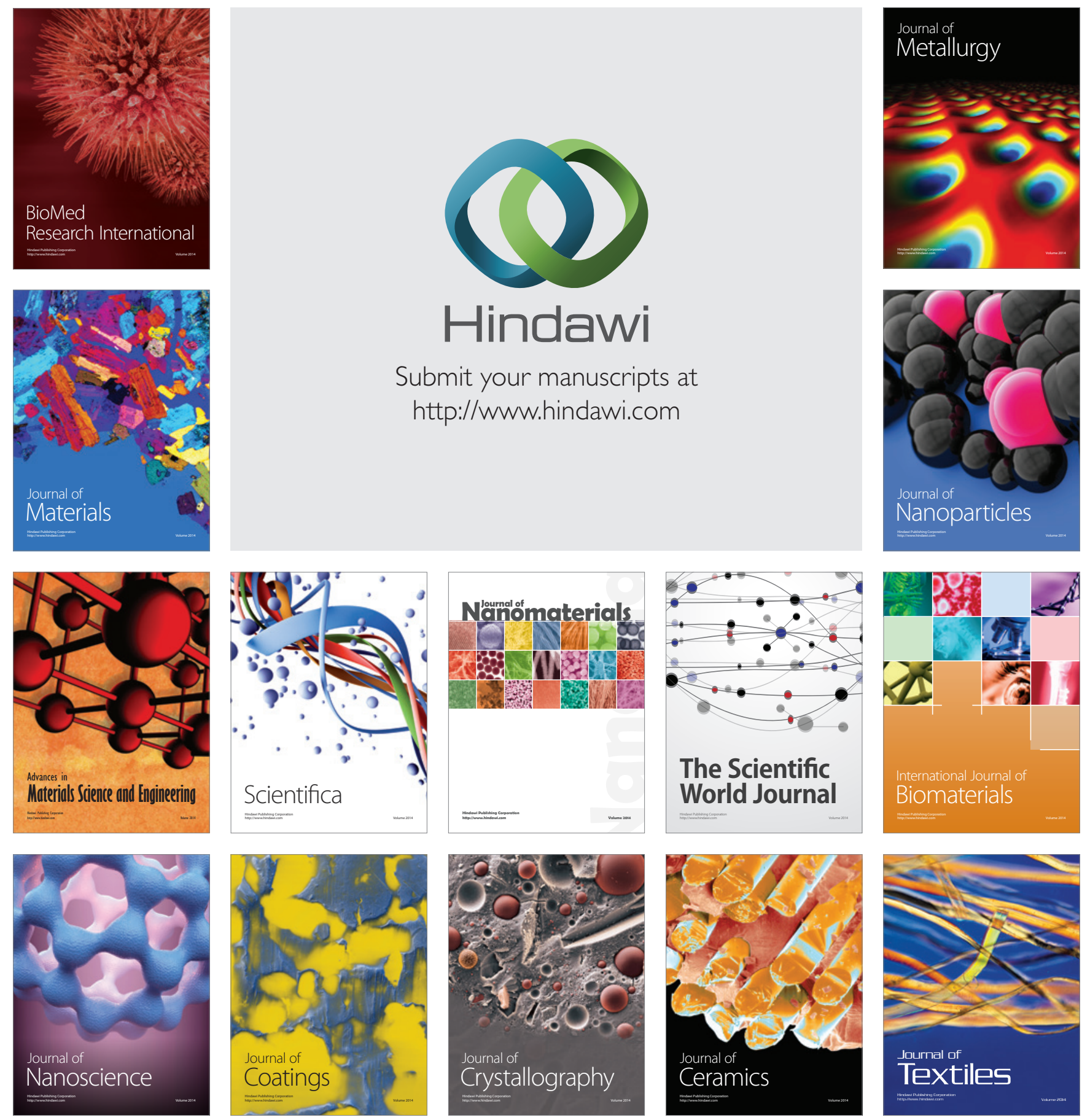